\title{
Linx
}

Revue des linguistes de l'université Paris X Nanterre

$11 \mid 1999$

Typologie des langues, universaux linguistiques

\section{Description linguistique et grammaire universelle : réflexions sur la notion de finitude à partir de la grammaire du wolof}

Anne Zribi-Hertz et Lamine Diagne

\section{OpenEdition}

Journals

Édition électronique

URL : http://journals.openedition.org/linx/940

DOI : 10.4000/linx.940

ISSN : 2118-9692

Éditeur

Presses universitaires de Paris Nanterre

Édition imprimée

Date de publication : 1 décembre 1999

Pagination : 205-215

ISSN : 0246-8743

\section{Référence électronique}

Anne Zribi-Hertz et Lamine Diagne, « Description linguistique et grammaire universelle : réflexions sur la notion de finitude à partir de la grammaire du wolof », Linx [En ligne], 11 | 1999, mis en ligne le 02 juillet 2012, consulté le 19 avril 2019. URL : http://journals.openedition.org/linx/940; DOI : 10.4000/ $\operatorname{linx} .940$

Ce document a été généré automatiquement le 19 avril 2019

Département de Sciences du langage, Université Paris Ouest 


\title{
Description linguistique et grammaire universelle : réflexions sur la notion de finitude à partir de la grammaire du wolof ${ }^{1}$
}

\author{
Anne Zribi-Hertz et Lamine Diagne
}

\section{Introduction}

Il est courant d'opposer les propositions finies aux propositions non finies, typiquement représentées par les in-fini-tives. Divers auteurs, parmi lesquels Benveniste (1950/1966) puis Renault (1991) et Nash \& Rouveret (1996), ont tenté de préciser le contenu de la notion de finitude (anglais : finiteness). Deux ingrédients sont, par hypothèse, présents dans une proposition finie et absents d'une proposition non finie - la flexion personnelle, et le temps :

\begin{tabular}{|l|l|l|l|}
\hline$(1)$ & a. & Pierre pense qu'il est beau. & (complétive finie) \\
\hline & b. & Pierre pense être beau. & (complétive non finie) \\
\hline$(2)$ & a. & Pierre regarde Marie qui danse le zouc. & (construction relative finie) \\
\hline \hline & b. & Pierre regarde Marie danser le zouc. & (construction infinitive) \\
\hline$(3)$ & a. & Comme il est guéri, Pierre a repris son travail. & (circonstancielle finie) \\
\hline & b. & Etant guéri, Pierre a repris son travail. & (circonstancielle non finie) \\
\hline
\end{tabular}


2 Le verbe des propositions finies (1a), (2a), (3a) est fléchi pour la personne et proprement spécifié pour le temps (l'opposition [ \pm passé]) ; celui des propositions non finies (1b), (2b), (3b) n'est spécifié ni pour l'un ni pour l'autre de ces deux traits. Nash \& Rouveret font l'hypothèse que le temps et la personne sont respectivement les manifestations verbale, et nominale, d'une même catégorie - la Finitude. Considérant ici les données du wolof, nous montrerons que cette langue nous invite à distinguer une catégorie Finitude distincte à la fois de la Personne et du Temps. Plus précisément, le wolof suggère que la Finitude ne se confond ni avec la Personne, ni avec le Temps, bien qu'il puisse exister certaines dépendances entre ces trois catégories.

\section{Constituants de la proposition wolof : premier survol}

Commençons par considérer des propositions wolof indépendantes, temporalisées, neutres (sans focalisation ni topicalisation), pour en dégager les constituants :

\begin{tabular}{|l|l|l|l|l|l|l|l|}
\hline (4a) & xale & $-y i$ & lekk & -oon & $-n a$ & $-\tilde{n u}$ & ceeb. \\
\hline & enfant & DFpl & manger & + pas & na & $3 \mathrm{pl}$ & riz \\
\hline
\end{tabular}

\begin{tabular}{|l|l|l|l|l|l|l|}
\hline$(4 \mathrm{~b})$ & {$[\varnothing]$} & lekk & - oon & $-n a$ & $-\tilde{n} u$ & ceeb. \\
\hline & & manger & + pas & na & $3 \mathrm{pl}$ & riz \\
\hline & \multicolumn{6}{|l}{} \\
\hline
\end{tabular}

4 L'ordre des constituants est SVO. Le radical du verbe (lekk, 'manger') porte un affixe de temps (-oon, +passé), un affixe laissé ici non glosé, $\underline{- \text { na }}$, et une désinence de personne (-ñu, $3 \mathrm{pl}$ ). Le sujet anaphorique ('ils') est réalisé à gauche du verbe fléchi comme un pronom nul. Dans l'exemple (5), la flexion personnelle a changé (1sg) :

\begin{tabular}{|l|l|l|l|l|l|l|l|}
\hline$(5)$ & {$[\varnothing]$} & Lekk & - oon & $-n a$ & $-a$ & ceeb. \\
\hline & & Manger & +pas & na & 1 sg & riz \\
\hline
\end{tabular}

5 L'affixe -na est invariable quelle que soit la personne. En (6), le temps [+passé] est remplacé par le temps [-passé] :

\begin{tabular}{|l|l|l|l|l|l|l|l|l|}
\hline$(6 a)$. & xale & $-y i$ & lekk & $\varnothing$ & $-n a$ & $-\tilde{n} u$ & ceeb. & \\
\hline
\end{tabular}




\begin{tabular}{|c|c|c|c|c|c|c|}
\hline enfant & DFpl & manger & -pas & na & $3 \mathrm{pl}$ & riz \\
\hline \multicolumn{7}{|c|}{ 'Les enfants ont mangé du riz.' } \\
\hline
\end{tabular}

\begin{tabular}{|l||l|l|l|l|l|l|}
\hline$(6 \mathrm{~b})$ & {$[\varnothing]$} & lekk & $\varnothing$ & $-n a$ & $-a$ & ceeb. \\
\hline & & Manger & -pas & na & $1 \mathrm{sg}$ & riz \\
\hline & \multicolumn{1}{|l}{} \\
\hline
\end{tabular}

6 Toutes les phrases qui précèdent sont traduites en français à l'aspect accompli ('ils avaient mangé', 'j'ai mangé'). L'interprétation accomplie, ou plus précisément, nonimperfective, est en effet la lecture non marquée avec un verbe processif comme 'manger' (cf. Robert 1994). Les interprétations imperfectives sont obtenues en insérant dans la flexion verbale l'auxiliaire imperfectif $\underline{\text { di : }}$

\begin{tabular}{|l|l|l|l|l|l|l|l|l|}
\hline (7a) & Xale & -yi & d(i) & -oon & -na & -ñu & lekk & ceeb. \\
\hline & enfant & DFpl & +ipf & +pas & na & 3pl & manger & riz \\
\hline
\end{tabular}

\begin{tabular}{|l|l|l|l|l|l|l|l|l|}
\hline$(7 \mathrm{~b})$ & xale & $-y i$ & $d i$ & $\varnothing$ & $-n a$ & $-\tilde{n u}$ & lekk & ceeb. \\
\hline \hline & enfant & DFpl & +ipf & - pas & na & $3 \mathrm{pl}$ & manger & riz \\
\hline \hline
\end{tabular}

7 C'est ici l'auxiliaire di, et non plus le verbe lexical, qui porte les affixes flexionnels Temps, -na, et Personne: cette situation est assez comparable à celle qu'on observe dans les propositions auxiliées du français.

8 Tous les exemples qui précèdent sont des phrases affirmatives. La négation, quand elle se manifeste, est, dans ce type de phrases, intégrée à la flexion verbale et précède la désinence personnelle dans l'ordre linéaire :

\begin{tabular}{|l|l|l|l|l|}
\hline (8a) & xale & $-y i$ & lekk-na-ñu & ceeb. \\
\hline & \multicolumn{3}{|l}{ 'Les enfants ont mangé du riz.' } \\
\hline
\end{tabular}

\begin{tabular}{|l|l|l|l|l|}
\hline$(8 b)$ & $x a l e$ & $-y i$ & lekk-u-ñu & ceeb. \\
\hline & 'Les enfants n'ont pas mangé de riz.' \\
\hline
\end{tabular}


Cette paire de formes suggère, incorrectement, que l'affixe -na pourrait marquer la polarité affirmative (analyse envisagée par Dunigan 1994). On constate toutefois que la négation apparaît dans divers contextes dont -na est exclu, par exemple :

\begin{tabular}{|l|l|l|l|l|l|l|l|l|}
\hline (9a) & xale & $-y i$ & $-a$ & lekk & $\left({ }^{*}-n a\right)$ & ceeb. & & {$[y i-a>y a-a]$} \\
\hline \hline & enfant & DFpl & foc & Manger & & riz & & \\
\hline \hline
\end{tabular}

\begin{tabular}{|l|l|l|l|l|l|l|}
\hline$(9 \mathrm{~b})$ & zale & $-y i$ & $-a$ & lekk & $-u l$ & ceeb. $^{3}$ \\
\hline \hline & enfant & DFpl & foc & manger & +neg & riz \\
\hline
\end{tabular}

10 L'affixe -na n'apparait jamais dans les phrases focalisées comme (9), qui permettent en revanche l'occurrence de la négation. Si -na était un marqueur de polarité affirmative, on s'attendrait à ce qu'il puisse apparaître en (9a), contrastant avec (9b). Le fait que tel ne soit pas le cas indique que -na est indépendant de la polarité, et que l'absence de -na en présence de la négation en (8b) doit être expliquée par une contrainte séparée.

11 Nous supposons donc que les propositions wolof illustrées en (4), (5), (6) et (7) et (8), comportent cinq spécifications flexionnelles, dont l'une reste à identifier :

\begin{tabular}{|c|c|}
\hline [區 aspect & $(+\mathrm{ipf}=\underline{\mathrm{di}},-\mathrm{ipf}=\varnothing)$ \\
\hline [橉 temps & $(+$ pas $=\underline{- \text { oon }},-$ pas $=\varnothing)$ \\
\hline 㷂 polarité & $(+$ nég $=\underline{-u(1)},-$ neg $=\varnothing)$ \\
\hline [國] NA & (à identifier) \\
\hline 嗬 personne & \\
\hline
\end{tabular}

12 Dans l'optique de la théorie de l'incorporation de Baker (1988), on peut concevoir ces cinq catégories comme autant de projections fonctionnelles dans lesquelles le verbe lexical ou l'auxiliaire monte successivement pour s'attacher la flexion : 
(10) dérivation des formes verbales fléchies

Personne (3pl : - $\tilde{n} u$ )

$t$ NA

¿ polarité (-nég:ø)

$\tau$ temps (+pas:-oon)

$\tau$ aspect (-ipf: $:$ )

t'verbe (lekk)

lekk-ø-oon-ø-na-ñu
Personne (3pl : - $-\tilde{n} u$ )

t NA

¿ polarité (-nég: ø)

$\tau$ temps (+pas:-oon)

$\tau$ aspect (+ipf : di)

$\tau$ verbe (lekk)

d(i)-oon-e-na-ñu lekk

\section{Distribution du morphème -na}

L'analyse en constituants a déjà révélé que -na est indépendant de la personne, de la polarité, du temps et de l'aspect. Dans tous les cas où il apparaitt, $\underline{-n a}$ est obligatoire. Ainsi est-il interdit de le supprimer en (11) :

\begin{tabular}{|l|l||l|l|l|l|l|l|l|l|}
\hline$(11)$ & $x a l e$ & $-y i$ & lekk & $\varnothing$ & -oon & $\varnothing$ & $*(-n a)$ & $-\tilde{n} u$ & ceeb \\
\hline
\end{tabular}

14 Toutefois, -na n'apparaît pas dans toute proposition, en wolof. Nous avons déjà vu qu'il est absent d'une phrase négative, même quand celle-ci est indépendante et fléchie pour la personne (cf. (8b)). L'affixe -na est par ailleurs absent d'une proposition à argument (sujet) focalisé comme (9a), et d'une relative, cf. (12):

\begin{tabular}{|l|l|l|l|l|l|l|l|l||l|}
\hline$(12 \mathrm{a})$ & $c e e b$ & $-b i$ & $x a l e$ & $-y i$ & lekk & $\varnothing$ & -oon & $\emptyset$ & $\left({ }^{*}-n a\right)$ \\
\hline & riz & DFsg & enfant & DFpl & manger & -ipf & +pas & -neg & \\
\hline \hline
\end{tabular}

\begin{tabular}{|l|l|l|l|l|l|l|l|l|l|}
\hline$(12 b)$ & $c e e b$ & $-b i$ & $x a l e$ & $-y i$ & lekk & $\varnothing$ & $\varnothing$ & $\varnothing$ & $\left({ }^{*}-n a\right)$ \\
\hline \hline & riz & DFsg & enfant & DFpl & manger & -ipf & -pas & -neg & \\
\hline \hline
\end{tabular}

\begin{tabular}{|l|l|l|l|l|l|l|l|l|l|}
\hline$(12 \mathrm{c})$ & ceeb & $-b i$ & xale & $-y i$ & lekk & $\varnothing$ & $\varnothing$ & -ul & $\left({ }^{*}-n a\right)$ \\
\hline & riz & DFsg & enfant & DFpl & manger & -ipf & -pas & +neg & \\
\hline \multicolumn{8}{|l}{} \\
\hline
\end{tabular}


L'affixe -na est également absent de la proposition enchâssée de (13), que nous nommerons infinitive :

\begin{tabular}{|l|l|l|l|l|l|l|l|l|}
\hline$(13)$ & Aram & gis-na-ø & [ngeen & lekk & $\emptyset$ & $\varnothing$ & $\emptyset$ & $\left({ }^{*}\right.$-na)ceeb]. \\
\hline & Aram & avu & $2 \mathrm{pl}$ & manger & -ipf & -pas & -neg & riz \\
\hline
\end{tabular}

La relative (12) et l'infinitive (13) sont spécifiées pour l'aspect, le temps et la polarité. Toutefois, le temps de la relative est libre, tandis que celui de l'infinitive est dépendant du temps-matrice :

\begin{tabular}{|l|l|l|l|l|l|l|l|l|l|}
\hline (14a) & Aram & gis & -oon -na-ø & [ngeen & lekk & $\varnothing$ & -oon & $\varnothing$ & ceeb]. \\
\hline \hline & Aram & voir & +pas NA 3sg & $2 \mathrm{pl}$ & manger & -ipf & +pas & -neg & riz \\
\hline
\end{tabular}

\begin{tabular}{|l|l|l|l|l|l|l|l|l|l|l|}
\hline$(14 \mathrm{~b})$ & *Aram & gis & $\underline{\emptyset}$ & -na-ø & [ngeen & lekk & $\varnothing$ & -oon & $\varnothing$ & ceeb]. \\
\hline & Aram & voir & -pas & NA 3sg & 2 pl & manger & -ipf & +pas & -neg & riz \\
\hline
\end{tabular}

17 Le verbe de l'infinitive et de la relative ne porte pas l'affixe -na, et n'est pas fléchi pour la personne. Ces deux propriétés corrélées caractérisent aussi le verbe des propositions à argument focalisé, comme (9a).

Les données examinées suggèrent donc une dépendance asymétrique entre l'affixe -na et la flexion personnelle : si -na apparaît sur le verbe ou l'auxiliaire, il sert de support à la flexion personnelle. Le seul cas où le morphème na ne supporte pas la flexion personnelle est celui où il n'est pas affixé au verbe, mais initial dans sa proposition, comme en (15) :

\begin{tabular}{|l|l|l|l|l|l|}
\hline$(15)$ & na & xale & $-y i$ & lekk & ceeb! \\
\hline & na & enfant & DFpl & manger & riz \\
\hline & \multicolumn{5}{|l}{ 'Il faudrait que les enfants mangent du riz!' } \\
\hline
\end{tabular}

Dans cette construction, l'absence de flexion personnelle est corrélée au fait que les constituants majeurs de la prédication (sujet et prédicat) restent à droite de -na, et ne créent donc pas de configuration d'accord dont la désinence personnelle serait l'épel.

Au terme de ces remarques nous pouvons avancer les hypothèses suivantes : 
\begin{tabular}{|l|l|}
\hline (16a) & $\begin{array}{l}\text { Personne est une excroissance (une 'proxy' : cf. Nash \& Rouveret 1996) de la catégorie } \\
\text { épelée par -na dans les propositions affirmatives. }\end{array}$ \\
\hline (16b) & $\begin{array}{l}\text { La tête Personne permet d'identifier le sujet nul, et d'accorder le prédicat verbal avec le } \\
\text { sujet plein déplacé dans son spécificateur. }\end{array}$ \\
\hline
\end{tabular}

21 Il découle de (16b) que la flexion personnelle est absente des phrases obligatives comme (15), qui ne satisfont pas la configuration d'accord. Il découle de (16a) que la flexion personnelle est crucialement solidaire d'une autre catégorie, épelée par -na, qu'il nous reste à identifier de façon précise - soit $\mathrm{K}$. Nous avons déjà vu que $\mathrm{K}$ est indépendante $\mathrm{du}$ temps, de l'aspect et de la polarité. La formulation (16c) nous oblige à supposer que dans une proposition négative comme (8b), qui est fléchie pour la personne, $\mathrm{K}$ est présente en syntaxe mais phonétiquement nulle.

\section{La projection Finitude}

Notre inventaire des types de propositions, en wolof, doit être enrichi pour intégrer les exemples comme (17) :

\begin{tabular}{|l|l|l|l|l|l|l||l|l|l|l|}
\hline$(17)$ & $B i$ & $x a l e$ & $-y i$ & lekk & $\varnothing$ & $\varnothing$ & $\varnothing$ & -ée & ceeb & -bi, \\
\hline & quand & enfant & DFpl & manger & -ipf & -pas & -neg & EE & riz & DFsg \\
\hline
\end{tabular}

\begin{tabular}{|l|l|l|l|l|l|l|l|}
\hline jigéen & -ñi & dem & $\varnothing$ & $\emptyset$ & $\varnothing$ & -na & -ñu. \\
\hline femme & DFpl & partir & -ipf & -pas & -neg & na & $3 \mathrm{pl}$ \\
\hline
\end{tabular}

Cette phrase complexe est composée de deux propositions, dont la première contient un affixe flexionnel non rencontré jusqu'ici : -ée. Cet affixe se manifeste linéairement à la périphérie droite du verbe ou de l'auxiliaire fléchi, dans certaines subordonnées. Le verbe qui porte l'affixe -ée en (17) est fléchi pour l'aspect, le temps et la polarité, mais non pour la personne. L'affixe -ée n'intervient que dans des subordonnées d'un certain type, introduites par un morphème monosyllabique traduit en français par 'quand', 'pendant que' , 'si' ou 'comme'. ${ }^{4}$ Dans l'ordre linéaire, ces subordonnées semblent généralement précéder la principale dont elles dépendent ; sémantiquement, elles délimitent un espace temporel distinct de Т0 (T0 = temps de l'énonciation), et dont l'interprétation de la principale est étroitement solidaire.

L'ordre linéaire des morphèmes suggère que l'affixe -ée occupe la même position structurale que l'affixe -na, dont il est complémentaire. Les affixes -na et -ée sont l'un et l'autre indépendants de l'aspect, du temps et de la polarité . Ils se distinguent l'un de l'autre par le fait que -na (mais non -ée) sert de support à la flexion personnelle, et par le fait que -ée (mais non-na) marque nécessairement un espace temporel distinct de T0. 
Pour réunir ces différents ingrédients de la description, nous proposerons les hypothèses (18) :

\begin{tabular}{|l|l|}
\hline$(18 \mathrm{a})$ & $\begin{array}{l}\text { Certaines propositions wolof incluent dans leur représentation structurale une } \\
\text { projection Finitude, placée au-dessus de la Polarité et au-dessous de la Personne. }\end{array}$ \\
\hline$(18 \mathrm{~b})$ & La tête Finitude est le lieu où l'énonciateur (E) est encodé dans la structure. \\
\hline$(18 \mathrm{c})$ & L'énonciateur peut être spécifié positivement (+E) ou négativement (-E). \\
\hline$(18 \mathrm{~d})$ & $\begin{array}{l}\text { Les affixes -na et -ée épellent respectivement les valeurs positive et négative du trait }[ \pm \mathrm{E}], \\
\text { dans la tête Finitude. }\end{array}$ \\
\hline
\end{tabular}

En vertu de (18a), toute proposition wolof n'est pas spécifiée pour le trait [ $\pm E]$ - autrement dit, n'encode pas l'énonciateur dans sa représentation syntaxique. Les propositions qui ne sont pas spécifiées pour ce trait n'ont pas de projection Finitude - ce sont donc des propositions NON finies : elles incluent notamment, en wolof, les propositions à argument focalisé, les relatives, et les 'infinitives' comme (13). Les propositions finies, formellement caractérisées par leur projection Finitude, peuvent être spécifiées positivement ou négativement pour le trait $[ \pm \mathrm{E}]$ : la valeur positive $[+\mathrm{E}]$, généralement épelée par $-n a^{5}$, caractérise les propositions indépendantes 'neutres' (sans argument focalisé), directement interprétées par rapport à T0; le trait [-E], épelé par -ée, caractérise des propositions subordonnées délimitant un espace temporel distinct de T0.

Notre analyse du morphème na est confirmée par le contraste, noté par Diouf (1985: 48) entre les constructions impérative (19a) et obligative (19b), qui s'emploient toutes deux pour énoncer un ordre mais se distinguent l'une de l'autre par l'occurrence de na dans la proposition obligative :

\begin{tabular}{|l|l|l|l|}
\hline$(19 a)$ & dem & -al- $\varnothing$ & tool ! \\
\hline & aller & IMPsg & champ \\
\hline & 'Va au champ!' \\
\hline
\end{tabular}

\begin{tabular}{|l|l|l|l|l|}
\hline$(19 \mathrm{~b})$ & $n a$ & $n g a$ & dem & tool! \\
\hline & $+\mathrm{E}$ & $2 \mathrm{sg}$ & aller & champ \\
\hline & 'Tu iras au champ, c'est mon désir, je te le suggère !' \\
\hline
\end{tabular}

Les traductions de Diouf (1985), reproduites ci-dessus, suggèrent que na encode bien l'énonciateur dans la phrase obligative.

Dialo (1981) et Ka (1982) ont proposé d'étiqueter 'énonciatives' les constructions en na, que Fal (1991) a, de son côté, envisagé d'appeler 'terminatives'. Notre analyse de na 
comme l'épel positif du trait 'Enonciateur' dans la tête 'Finitude' formalise exactement cette double intuition des linguistes sénégalais.

\section{Conclusion} structure fonctionnelle de la proposition inclut, dans cette langue, une projection que nous étiquetons Finitude, dans laquelle est encodé l'énonciateur. L'encodage peut être positif (+E) ou négatif (-E) : dans le premier cas, la proposition est directement interprétée par rapport à $\mathrm{T} 0$; dans le second cas, un espace temporel distinct de T0 délimite l'interprétation. L'énonciateur peut aussi ne pas être encodé du tout dans la structure de la proposition : dans ce cas, la Finitude n'est pas projetée, et le trait $[ \pm E]$ n'est pas spécifié.

(1981), Diou (1985) ou Robert (1994), font valoir que l'énonciateur est toujours implicitement présent derrière un énoncé, quelle qu'en soit la forme. Nous admettons volontiers cette idée générale, mais nous prétendons qu'il est souhaitable de distinguer les perspectives pragmatique et syntaxique : s'il est vrai que tout énoncé émane d'un énonciateur, il n'est pas vrai que l'énonciateur soit encodé dans toute construction - nous nous rallions notamment sur ce point à Benveniste (1946/1966) et Banfield (1982).

Nos résultats nous invitent par ailleurs à mettre en question la conception binaire de la finitude évoquée dans l'introduction, selon laquelle une proposition finie s'opposerait simplement à une proposition 'non finie'. Selon nos hypothèses, une proposition est finie si l'énonciateur est encodé - positivement ou négativement - dans sa structure. Une proposition [+E], spécifiée positivement pour la finitude, s'oppose donc, d'une part, à une proposition [-E], spécifiée négativement pour la finitude, et d'autre part, à une proposition [-finie], c'est-à-dire non spécifiée pour la finitude : en wolof, les propositions temporelles sont [-E], tandis que les propositions 'infinitives', relatives, impératives, ou à argument focalisé, sont [-finies].

La notion de finitude, telle que nous la définissons en observant le wolof, se révèle indépendante de l'idée d'« autonomie », puisqu'une proposition peut être indépendante et non finie (propositions à argument focalisé) et qu'il existe aussi des propositions finies dépendantes (subordonnées temporelles). La finitude entretient par ailleurs des relations différentes avec le Temps, et avec la Personne. Le trait [+E], qui incarne la finitude positive, se révèle bien être un préalable nécessaire à l'occurrence de la flexion personnelle. En revanche, l'opposition temporelle [tpassé] semble largement indépendante $\mathrm{du}$ trait $[+\mathrm{E}]$ en wolof, puisqu'elle est présente aussi bien dans les propositions [-E] (subordonnées temporelles) que dans certaines propositions [-finies] (relatives, argument focalisé). Le wolof contraste ici de façon frappante avec le français, dont les propositions [-finies] sont, typiquement, temporellement déficientes. Le wolof et le français se rejoignent aux deux pôles de la Finitude: dans les deux langues, les propositions à temps indépendant et à flexion personnelle sont [+finies], tandis que les propositions sans flexion personnelle et à temps déficient ou dépendant sont [-finies]. Une variation intéressante s'observe pour les cas intermédiaires: les propositions relatives et focalisées sont [-finies] en wolof, tandis que les relatives et clivées du français semblent, jusqu'à preuve du contraire, [+finies]; les propositions [-E] du wolof ne semblent pas avoir d'équivalent en français, tandis que les propositions subjonctives 
(+personne, -temps) du français moderne n'ont pas de contrepartie en wolof ; les propositions aoristiques du wolof (temps dit 'narratif') sont [-finies], celles du français (passé simple) semblent plutôt 'faiblement finies' (déficientes pour la deuxième personne, cf. Banfield 1982). Tous ces contrastes mériteraient d'être étudiés avec le plus grand soin.

\section{BIBLIOGRAPHIE}

BAKER, M. (1988) Incorporation. Chicago : Chicago University Press.

BANFIELD, A. (1982) Unspeakable sentences. Londres : Routledge.

BENVENISTE, E. (1946), 'Structure des relations de personne dans le verbe', repris dans Benveniste (1966).

BENVENISTE, E. (1950) ‘La phrase nominale’, repris dans Benveniste (1966).

BenVeniste, E. (1966) Problèmes de linguistique générale. Paris : Gallimard.

DiALO, A. (1981) Structures verbales du wolof contemporain. Université de Dakar : C.L.A.D.

Diouf, J.L. (1985) Introduction à une étude du système verbal wolof: relation modes, pronoms sujets et autres modalités du prédicat. Université de Dakar : C.L.A.D.

Dunigan, M. (1994) The clausal structure of Wolof : a study of focus and cliticization. Thèse inédite. Université de Caroline du Nord, Chapel Hill.

FAL, A. (1991) Alphabétisation en wolof. Guide orthographique. Dakar.

KA, O. (1982) La syntaxe du wolof : essai d'analyse distributionnelle. Thèse de doctorat inédite, Université de Dakar.

NASH, L. et Rouveret, A. (1996) “Proxy categories and Phrase Structure Theory”. Proceedings of NELS 27.

NJIE, C. M. (1982) Description syntaxique du wolof de Gambie. Dakar : Les Nouvelles Editions Africaines.

RENAUlt, R. (1991) Recherches en syntaxe du finnois : les désinences personnelles. Thèse de doctorat, Université Paris-8.

ROBERT, S. (1994) Approche énonciative du système verbal. Le cas du wolof. Paris : CNRS.

SAUVAGEot, S. (1965) Description synchronique d'un dialecte wolof: le parler du Dyolof. Dakar : I.F.A.N.

YAGUello, M. (1981) Alice au pays du langage : pour comprendre la linguistique. Paris : Seuil.

\section{NOTES}

1. Nous avons une dette envers J. Guéron, M. Kaneko, A. Kihm, L. Nash et R. Renault et A. Rouveret, qui ont attiré notre attention sur certains points importants et sur certaines bêtises à ne pas dire. 
2. La traduction du présent ferait appel à un autre type de construction (copulative), que nous laissons de côté dans cette brève présentation.

3. La consonne finale de l'affixe négatif [-ul] est tronquée lorsque celui-ci sert de support à un affixe à initiale consonantique, cf. (8b).

4. La traduction dépendra de la forme du morphème introducteur $(\mathrm{bi} / \mathrm{ba} / \mathrm{bu} / \mathrm{su} / \mathrm{ni})$ et de la spécification aspectuelle (+ipf ou -ipf).

5. Le trait $[+E]$ est appauvri, donc non épelé, dans les phrases négatives comme (8b), ainsi que dans les propositions que Robert (1994) appelle 'à emphase du verbe', dont nous ne traitons pas ici.

6. Dunigan (1994) nomme subjonctives les propositions 'narratives' du wolof (aoristiques), les subordonnées temporelles en -ée (morphème qu'elle glose par [+irrealis] et que nous glosons par [-E]), ainsi que les subordonnées que nous appelons plus haut 'infinitives'. Ces deux dernières classes de propositions se caractérisent par le fait qu'elles sont marquées comme dépendantes par rapport à la principale (les infinitives, par la concordance des temps ; les temporelles, par l'affixe -ée) ; mais elles se distinguent crucialement des subjonctives du français par l'absence de flexion personnelle et, pour les temporelles, par le fait qu'elles sont proprement spécifiées pour le temps. Les propositions aoristiques (narratives) du wolof partagent avec les subjonctives du français leur déficience temporelle, mais s'en distinguent radicalement par leur non-dépendance.

\section{AUTEURS}

ANNE ZRIBI-HERTZ

UPRESA 7023, Paris-8/CNRS

\section{LAMINE DIAGNE}

UPRESA 7023, Paris-8/CNRS 